\title{
Salud bucal: curricularización de la extensión en los Espacios de Formación Integrados de la Universidad Nacional de Córdoba
}

\author{
Natalia Agüero \\ natucha2111@hotmail.com
}

\section{Leonor Lattanzi}

leonorll|@hotmail.com

Lila Susana Cornejo

lisucor@gmail.com

\author{
Pablo Cristian Gigena \\ pablo_gigena@hotmail.com
}

Docentes investigadores de la

Universidad Nacional de Córdoba,

Argentina.
Integración de la docencia y la extensión /

Intervenciones

RECEPCIÓN: 24/06/16

ACEPTACIÓN FINAL: 10/10/16

\section{Resumen}

Se propone la curricularización de la extensión universitaria en Espacios de Formación Integrados en el proyecto "Desarrollo e implementación de estrategias de promoción de la salud contextualizadas para la apropiación de conductas de salud bucal en adolescentes de comunidades educativas vulnerables", desarrollado desde la Facultad de Odontología y Escuela de Trabajo Social de la Universidad Nacional de Córdoba en la comunidad educativa del Instituto Provincial de Educación Media n 377 de Barrio Ciudad Villa Angelelli. Los estudiantes de esta escuela, familias y comunidad barrial, se caracterizan por vivir situaciones con vulnerabilización socioambiental. En prácticas de extensión integradas al currículo universitario, los estudiantes se involucran en la resolución de problemáticas de la comunidad donde se ponen a prueba conocimiento académico, interdisciplina y pluralidad de profesiones en interacción con otros actores sociales. Se problematizan y resignifican las prácticas de enseñanza, lo cual posibilita formas de aprender y de evaluar procesos para una formación más integral de futuros profesionales universitarios.

Palabras-clave

- Curricularización de la extensión

- Clínica del sujeto

- Salud bucal

- Juventud

- Interdisciplina

\section{Resumo}

Propõe-se a curricularização da extensão universitária em Espaços de Formação Integrados no projeto "Desenvolvimento e implementação de estratégias de promoção da saúde contextualizadas para a apropriação de condutas de saúde bucal em adolescentes de comunidades educativas vulneráveis", desenvolvido pela Faculdade de Odontologia e Escola de Trabalho Social da Universidade Nacional de Córdoba, na comunidade educativa Instituto Provincial de Educação Secundária $N^{\circ} 377$ do Bairro Ciudad Villa Angelelli. Os alunos desta escola, famílias e comunidade do bairro caraterizam-se por viver situações com vulnerabilidade social e ambiental. Em práticas de extensão integradas ao currículo universitário, os alunos se envolvem na resolução de problemáticas da comunidade, onde são testados o conhecimento acadêmico, a interdisciplinaridade e a pluralidade de profissões em interação com outros atores sociais.

Problematizam-se e se ressignificam as práticas de ensino, possibilitando formas de aprender e de avaliar processos para uma formação mais integral de futuros profissionais universitários.

Palavras-chave

- Curricularização da extensão

- Clínica do indivíduo

- Saúde bucal

- Juventude

- Interdisciplinar
Para citación de este artículo

Agüero, N.; Lattanzi, L.; Cornejo, L. y Gigena, P. (2016).

Salud bucal: curricularización de la extensión en los Espacios de Formación Integrados de la Universidad Nacional de Córdoba. En Revista +E versión digital, (6), pp. 286-291. Santa Fe, Argentina: Ediciones UNL. 


\section{Introducción}

La curricularización de la extensión universitaria supone integrar contextos y contenidos curriculares, articular lógicas institucionales diversas, fomentar habilidades sociales en los estudiantes, incentivar el trabajo en equipo, reconocer y respetar las trayectorias y saberes de actores sociales involucrados en las prácticas con una mirada crítica sobre problemáticas sociales.

Desde la universidad pública, y en este caso desde la Facultad de Odontología y la Escuela de Trabajo Social de la Universidad Nacional de Córdoba, se presenta la experiencia que surge a través del proyecto de extensión denominado "Diagnóstico, diseño colectivo, implementación y evaluación de estrategias de promoción prevención de salud, en la comunidad educativa IPEM 377" Secretaría de Extensión Universitaria (SEU) de la Universidad Nacional de Córdoba (UNC), aprobado y subsidiado (Res. 114/14 SEU, UNC). Este proyecto se ha puesto en marcha en el ámbito de la comunidad educativa del Instituto Provincial de Educación Media (IPEM) n 377 de Barrio Ciudad Villa Angelelli desde 2014 a la fecha. Tiene como destinatarios directos a los jóvenes estudiantes (Dolto, 1990; Fize, 2001 y Escudero, 2008) que asisten a esa institución de enseñanza media y en forma indirecta a las familias de los adolescentes y la comunidad barrial de donde provienen Nuestro Hogar III, Villa Angelelli I y II y Villa Rivadavia, barrios considerados de alta vulnerabilidad socioambiental.

La experiencia se enfoca en el abordaje de algunas de las problemáticas que surgen de las condiciones de vulnerabilidad de los jóvenes y su contexto para generar un espacio reflexivo en la escuela sobre la importancia del cuidado de la salud en su componente bucal, haciendo hincapié en que la misma debe ser considerada desde una perspectiva integral, que reconoce las necesidades y con apoyo de las potencialidades de los jóvenes y de la población territorial.

El diagnóstico situacional efectuado reveló que los docentes, si bien manifiestan interés por la problemática de la salud bucal, cuentan con escasas estrategias pedagógicas para promoverla desde el aula. Respecto de los jóvenes, se evidenció un deterioro marcado de la situación clínico-odontológica, con mayor severidad en los estudiantes de los últimos años. Otro elemento que surgió fue la dificultad para acceder a la atención clínica odontológica restaurativa y de la promoción para la salud. A partir del reconocimiento de la incidencia que tiene la enfermedad bucal en la calidad de vida de los sujetos, se infiere la necesidad de profundizar el trabajo interdisciplinario, inicialmente con la generación de acciones educativas que promuevan la construcción de hábitos higiénico-dietéticos saludables pues, como se dijo, se observa un gran deterioro de los indicadores de salud bucal y con escasas posibilidades de resolverlo, para luego abordar estrategias emergentes propias del surgimiento de nuevas problemáticas sentidas por la comunidad, lo cual implica un diseño flexible del proyecto.
Se consideraron como antecedentes la experiencia de práctica académica de estudiantes y docentes de Trabajo Social durante varios años en instituciones de salud de esta zona relacionada con la intervención de éste con familias, las que ampliaron la posibilidad de continuidad desde la especificidad y lo interdisciplinario.

Los docentes de las distintas cátedras que formamos parte de esta experiencia sostenemos una organización que integra, aporta y capacita los recursos humanos tanto de la población como de los estudiantes que participan mediante la vinculación de la Universidad con las diferentes instituciones del medio a las que les interesan nuestras propuestas y garantizan su sustentabilidad.

\section{Los debates sobre la inserción curricular de la extensión} La universidad pública de Argentina, con el legado de la Reforma de 1918, consolidó tres funciones básicas: docencia, investigación y extensión, que se pensaron de manera integrada en pos de lograr una formación completa de los futuros profesionales que egresen de ella.

A lo largo de la historia, estas funciones fueron ocupando lugares diferentes y pensados desde concepciones distintas. Así, a finales del siglo XX se pudo observar que las universidades latinoamericanas fueron consolidando una tendencia a convertirse en una especie de "supermercados del conocimiento", con un sesgo profesionalista y credencialista, cristalizaron progresivamente una visión de esta institución como meramente "expendedora" de títulos y olvidaron otros aprendizajes que son parte sustancial de las instituciones de educación superior.

Se perdía de vista que:

"la calidad en las instituciones públicas está directamente vinculada con la preparación de la ciudadanía para desempeños políticos, profesionales, culturales, científicos, solidarios con el 'otro'. La universidad Latinoamericana democrática ayudó a entrenar a generaciones de jóvenes en una 'diversidad' de intereses que dinamizaron los espacios cívicos democráticos". (Mollis, 2003:203)

En ese contexto, la extensión ocupaba un lugar secundario o bien, a tono con las corrientes de mercantilización de la educación, se reducía a una "venta de servicios" de la universidad a empresas, instituciones u organizaciones comunitarias.

En los últimos años asistimos nuevamente a una revitalización del debate respecto de las funciones de la universidad, y particularmente del lugar de la extensión en los procesos de formación profesional, a partir de la iniciativa del Consejo Interuniversitario Nacional (CIN) en ocasión del tratamiento del tema del Compromiso Social en las Universidades Nacionales y de que se reuniera la documentación caracterizando la extensión en cada una ellas. Luego de un minucioso análisis y discusión sobre el tema en el plenario de Secretarios de Extensión llevado 
a cabo en la ciudad de San Salvador de Jujuy en agosto de 2011 (ULEU, 2011), se consensuó el siguiente concepto:

"Entendemos la extensión como espacio de cooperación entre la universidad y otros actores de la sociedad de la que es parte. Este ámbito debe contribuir al mejoramiento de la calidad de vida de las personas y está vinculado a la finalidad social de la Educación Superior: la democratización social, la justicia social y el derecho a la educación universal; se materializa a través de acciones concretas con organizaciones sociales, organizaciones gubernamentales y otras instituciones de la comunidad, desde perspectivas preferentemente multi e interdisciplinarias. Las acciones de extensión deberán desarrollarse desde un enfoque interactivo y dialógica entre los conocimientos científicos y los saberes, conocimientos y necesidades de la comunidad que participa. La extensión contribuye a la generación y articulación de nuevos conocimientos y nuevas prácticas sociales, integra las funciones de docencia e investigación, debe contribuir a la definición de la agenda de investigación y reflejarse en las prácticas curriculares". (CIN, 2012:5-6)

Un importante desafío que enfrentan las universidades es integrar las tres funciones básicas de la universidad, evitar la fragmentación entre las mismas y procurar su sistematicidad y su permanencia en el tiempo.

En el caso concreto de la integración entre la extensión y la docencia, se plantea que las universidades no deben orientar la formación solo con un sesgo "profesionalista" o meramente técnico, como señalábamos antes, sino que debe promover el desarrollo de capacidades que aporten a desenvolverse en un mundo cada vez más complejo y a su vez se plantea como objetivo central la formación de ciudadanos críticos y comprometidos socialmente.

"Sin lugar a dudas, para alcanzar estos objetivos, se requiere un importante esfuerzo académico e institucional que permita incorporar las diversas y complejas problemáticas sociales a los procesos de enseñanza y aprendizaje". (CIN, 2012:5)

Una de las propuestas que se ideó en pos de esta integración entre docencia y extensión es la inserción curricular de la función extensionista o "curricularización de la extensión". Esta propuesta plantea la incorporación de actividades con carácter extensionista al trayecto académico, a la formación profesional del estudiante universitario, otorgándole validez curricular a las mismas.

Al respecto, el CIN señala que las experiencias en desarrollo en este punto son escasas. Aunque existen propuestas concretas que están en proceso, son muy pocas las Universidades que han podido avanzar en propuestas en este sentido.

Según este organismo, en un relevamiento realizado se pueden identificar dos tipos de experiencias en curso: por un lado, el desarrollo de cursos — predominantemente optativos_- dirigidos a estudiantes de grado, con validez curricular, ya que se traducen en créditos del plan de estudios de la carrera que se cursa. Por otro lado, prácticas sociales que, fundamentadas y definidas desde su potencial formativo y desarrolladas bajo la responsabilidad de docentes y tutores, son incluidas con validez curricular y con carga horaria en el plan de estudios de la carrera de que se trate (CIN, 2012).

Este tipo de experiencias gira en torno a un dispositivo denominado Espacios de Formación Integral (EFI), "entendidos como actividades docentes que apuntan a la articulación de enseñanza, extensión e investigación en el proceso formativo de los estudiantes". (CIN, 2012:8)

Los EFI son concebidos como una de las alternativas para lograr un objetivo tan concreto como ambicioso pero digno de ser perseguido al vincular a estudiantes con diversas facetas de la problemática social y afinar sus capacidades no sólo para la reflexión crítica sino también, y sobre todo, para la construcción colectiva de propuestas que mejoren efectivamente la calidad de vida. Un punto de partida es esta definición de la extensión de Bordoli: "En sentido estricto, proceso dialógico y bidireccional, que busca la colaboración entre actores universitarios y actores sociales, en un pie de igualdad, dialogando y combinando sus respectivos saberes al servicio de objetivos socialmente valiosos, dando prioridad a los problemas de los sectores más postergados. En sentido amplio, la extensión comprende diversas formas de actividades en el medio social, tales como asistencia a la comunidad, difusión o divulgación científica y procesos de transferencia tecnológica. Estas actividades tienen un enfoque unidireccional y pretenden dar respuesta a demandas puntuales de la población. La extensión es una de las tres funciones básicas de la Universidad, junto con la enseñanza y la investigación". (2010:15)

\section{Característica académica de la práctica integrada}

En el contexto de las discusiones que se vienen dando en universidades públicas argentinas en torno a la elaboración de propuestas extensionistas con un marco de curricularización al interior de las funciones de extensión, hay coincidencia en cuanto a que esta inserción curricular de la extensión, articulada junto a la enseñanza y la investigación, requiere y promueve el diálogo de saberes y prácticas. Si bien esta visión transversal e integrada que proponemos en las distintas unidades académicas es baja, y por eso todavía predomina el trabajo vertical, compartimentado y muchas veces desarticulado.

La generación de distintas estrategias que permitan la implementación y curricularización de prácticas en espacios de formación integrados se proponen como dispositivos teóricoprácticos que combinen momentos de aprendizaje en el aula y en 
el terreno, donde docentes y estudiantes, interactuando con actores sociales, integren y articulen las tres funciones universitarias en un marco de diálogo de saberes y abordaje interdisciplinario.

Recientemente, en la Facultad de Odontología se logró la acreditación de horas en el espacio curricular de Práctica Profesional Supervisada, del $9^{\circ}$ cuatrimestre de la carrera en los estudiantes de Odontología y/o su acreditación en el espacio curricular de actividades optativas que el estudiante debe cumplimentar con validez curricular y con carga horaria en el plan de estudios de la carrera durante 2014/2015. Éste supone un primer avance y antecedente importante para lograr la inserción curricular de la extensión.

Respecto de los estudiantes de Trabajo Social, participan en el proyecto y realizan simultáneamente la práctica de intervención con familias. Este espacio facilita la apropiación, uso y transferencia de saberes, metodologías y técnicas que les permitirán construir, a lo largo del proceso académico y con gradual complejidad, el diseño de una propuesta de intervención con las familias de la zona. Prevé un trabajo en dos líneas en la búsqueda colectiva de soluciones a problemas seleccionados para su intervención: por un lado, acciones destinadas a la consolidación del equipo como grupo de referencia en la temática dentro de la Universidad promoviendo procesos de enseñanza y aprendizaje inclusivos e integrados, y por otro, aquellas acciones que permitan la conformación de las redes de vinculación interinstitucional y de abordaje interdisciplinario, promoviendo un trabajo participativo y en red desde un enfoque territorial.

\section{Nuestra experiencia...}

Desde la universidad pública y, en este caso, desde la Facultad de Odontología, se propone que la extensión y las prácticas integrales sean incorporadas. La curricularización de la Extensión supone pensar una estrategia integral, gradual y consensuada. Las prácticas clínicas, fundamentadas y definidas desde su potencial formativo y desarrolladas bajo la responsabilidad de docentes y tutores, son incluidas con validez curricular y con carga horaria en el plan de estudios de la carrera de Odontología desde la Práctica Profesional Supervisada. Presentamos una experiencia académica que, articulada con la comunidad donde trabajamos, nos impulsa a buscar respuestas, a exigir a los profesionales y potenciar capacidades para pensar y actuar en la transformación de la comunidad. Los criterios para resolver las problemáticas/ necesidades son el resultado de indagaciones y acuerdos entre investigadores docentes extensionistas, estudiantes extensionistas y la comunidad, y las propuestas responden a las necesidades de la vida cotidiana de los jóvenes y sus familias en tanto son trabajadas desde un espacio de identificación de potencialidades. Estas prácticas conjugan contenidos, disciplinas, saberes y funciones universitarias e intentan generar una continuidad de estos procesos a lo largo de la trayectoria formativa de los estudiantes. Promueven la iniciación al trabajo en grupo desde la perspectiva interdisciplinaria, por la que se vinculan servicios y áreas del conocimiento reunidos en una misma temática.

Nuestra propuesta es una construcción que rompe con la visión de las experiencias académicas donde los conocimientos y aprendizajes circulan al interior la misma universidad, que por lo común cierra los conocimientos científicos en procesos de producción y reproducción dentro de los recintos universitarios, y en este caso ofrece un dispositivo teórico y práctico integral e interdisciplinario y multiprofesional que intenta articular en el espacio áulico, pero fundamentalmente fuera de él, las funciones de enseñanza y de extensión. El aula deja de ser el único lugar donde se desarrollan los procesos de enseñanza y aprendizaje. La noción de espacio integra al aula pero también al trabajo de campo dentro de este proyecto de formación y de intervención. Consideramos que las prácticas académicas reorientadas y redimensionadas como tareas de extensión amplían su capacidad formativa al reflexionar sobre la calidad y la eficacia de los servicios brindados, abren espacios de discusión, sensibilización y formación, ya que contribuyen con acciones de servicios concretos y de interacción con la comunidad y ofrecen alternativas a ciertos problemas detectados en sus propios contextos de aplicación, alternativas que no podrán nunca estar desvinculadas del proceso de enseñanza-aprendizaje que las contiene y jerarquizan la relación entre la universidad y la comunidad.

Esta experiencia de extensión universitaria integrada opera a la vez como contexto de producción científica, retroalimenta la producción de conocimientos generales y profesionales, ejerce un efecto sinérgico importante al aprender mediante la creación de soluciones a las necesidades y/o expectativas de grupos sociales con quienes los estudiantes interactúan con objetivos iniciales de aprendizaje y, secundariamente, de brindar servicios profesionales. Es un flujo de racionalidades múltiples, donde las tres funciones de la universidad: la académica, de extensión y de investigación, se potencian unas a otras y contribuyen a fortalecer la formación de grado a través de su incorporación como actividad curricular de formación. Se trata de interpretar la extensión junto a la docencia y la investigación, en su sentido amplio, involucrándola en los más diversos aspectos con la sociedad y el medio, no sólo mediante la transferencia sino, y fundamentalmente, escuchando, aprendiendo, respetando "a los otros" como sujetos transformadores de sí mismos y de su entorno y como activos constructores de saberes sociales.

Es en este caso, detectar y atender los problemas de prácticas saludables de salud bucal que afectaban a los jóvenes y sus redes familiares, transmitir conocimientos para pensar y recrear caminos con ellos, avanzar hacia la superación de sus dificultades y mejorar 


\section{6}

no es sencillo trabajar desde esta perspectiva porque estamos

acostumbrados a procesos

tradicionales, específicamente

desde lo disciplinario

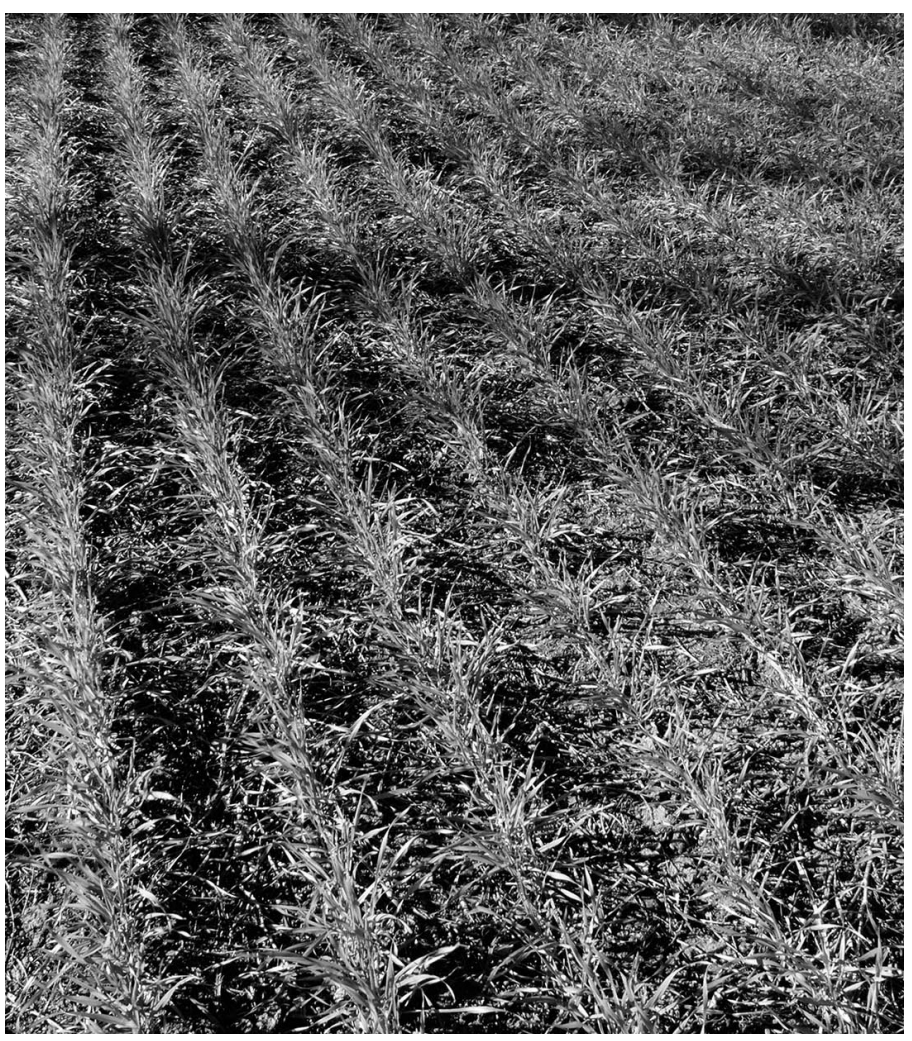

(c) Laura Hormaeche sus condiciones vitales en una lógica propositiva y proactiva, con un involucramiento de docentes estudiantes y actores sociales comunitarios, con la generación de espacios de vinculación e intercambios activos para la formulación conjunta de los problemas y la construcción de una respuesta integral a los mismos. Remite a un trabajo conjunto, que integra el protagonismo de los jóvenes, sus saberes, su cultura, sus habitus, sus capacidades de acción, donde todos, universitarios y no universitarios, aprenden y son portadores de saberes valiosos que nos interesa recuperar e incorporar y evitar una práctica académica aislada y cientificista. Los proyectos de extensión así entendidos pueden ser analizados desde una perspectiva que recupera la faz formativa, sea de los sujetos destinatarios de las acciones de extensión o de los sujetos universitarios encargados de motorizar dichas acciones, donde los enfoques pedagógicos y metodológicos desarrollados, las formas de construcción del conocimiento, la participación de los diferentes saberes y actores intervinientes son dimensiones inseparables. Prácticas formativas que estimulan capacidades críticas en el proceso de enseñanza-aprendizaje y renuevan el compromiso de la comunidad universitaria con la realidad social de la cual se nutre, forma parte, y en la que se desenvuelve.

\section{Conclusiones}

Comienza a desarrollarse una mayor conciencia universitaria sobre la necesidad de vincularse con la comunidad, de involucrarse con los problemas cotidianos y de trabajar al ritmo y con los tiempos que los problemas o las demandas requieren, para elaborar respuestas útiles y comprometidas, no sólo con el futuro sino con el presente, así como canalizar proyectos de importancia institucional y definir prioridades en función de un plan de trabajo integral. No es suficiente abrir las puertas de la universidad pública al medio, no alcanza con ofrecer lo que sabemos hacer ni con hacer lo que nos parece. Hoy la universidad debe hacer lo que es necesario. Es preciso salir y formar parte. El desafío es escuchar, integrar a la universidad con la sociedad e involucrarse.

Se requiere potenciar el vínculo entre la universidad y la sociedad y discutir dispositivos pedagógicos que interpelen las formas de enseñar y aprender, como el que proponemos, donde se integren 
las tres funciones de la universidad, para construir de manera conjunta y delimitar temáticas específicas en las cuales trabajar. Que signifiquen intercambios de saberes, respuestas integrales y nuevas maneras de consolidar proyectos para darles continuidad en el tiempo, más allá de los académicos.

Que la extensión deje de ser entendida como una actividad que se hace en un tiempo extracurricular, de manera aislada, como pasa hoy, que es una novedad curricularizar ciertas experiencias particulares y otorgarles créditos por el esfuerzo de algunos docentes sin que llegue a convertirse en una actividad de profundización trasversal y sostenida en todo el trayecto formativo de los estudiantes.

Supone un desafío para la universidad el hecho de incorporar la extensión universitaria al currículo y a cargo de especialistas que nos brinden fundamentaciones teóricas éticas y políticas para formar profesionales, que comprendan la complejidad de las transformaciones históricas, políticas, económicas, sociales, culturales acontecidas en nuestra sociedad y puedan aportar de manera reflexiva y crítica, desde las disciplina, sumándonos como profesiones junto a otros campos del saber en la interdisciplina y operativizar prácticas que favorezcan la calidad de vida de las comunidades.

No es sencillo trabajar desde esta perspectiva porque estamos acostumbrados a procesos tradicionales, específicamente desde lo disciplinario, a la independencia y seguridad que nos dan y donde nos movemos con cierta comodidad. Estas experiencias exigen la articulación entre diferentes ámbitos, facultades, cátedras, departamentos y servicios universitarios, para potenciar esfuerzos. Exige otro modo de administrar el tiempo y los espacios donde se desarrollan las actividades, ya que los procesos formativos son distintos según las características de cada grupo de estudiantes. La apuesta de estas prácticas y la de esta experiencia particular significan un aporte a la formación académica y profesional de los estudiantes. Los prepara para comprender la complejidad de las problemáticas sociales, para que tomen un papel activo frente a ellas y entiendan la función de la extensión como herramienta para promover el desarrollo local y regional.

Permite contextualizar histórica, social, económica, cultural y políticamente las situaciones abordadas, producir conocimientos desde el enfoque de Investigación Acción Participativa, ayuda al enriquecimiento de los conocimientos que se producen y reproducen, y valora el trabajo "interdisciplinario" y en equipo, de manera colaborativa.

Aporta, en definitiva, a formar profesionales críticos, preparados para trabajar con una diversidad de sujetos habilitados para diseñar propuestas de acción complejas e integrales ante las problemáticas actuales en la búsqueda de superar una formación profesional meramente técnica o profesionalista.

\section{Referencias bibliográficas}

Bordoli, E. (2010). Aportes para pensar la extensión universitaria. En: AA. VV. Extensión en obra. Experiencias, reflexiones, metodologías y abordajes en extensión universitaria, 13, 13-47. Consultado el 20/05/16 en: www.extension.edu.uy/ system/files_force/Extension_en_Obra_web.pdf\#page=13

CIN (2012). Acuerdo Plenario n 811/12. Plan estratégico de Extensión 2012-2015. Consultado el 20/02/16 en: http://www.unc.edu.ar/extension/fortalecimiento/documentos/Ac.PI\%20No\%20811-12.pdf

Dolto, F. (1990). La causa de los adolescentes. Barcelona: Seix Barral. Escudero, J.C. (2008). Proceso de integración del Sistema de Salud. Ciencias Sociales, 5, 121-131.

Fize, M. (2001). ¿Adolescencia en crisis? Buenos Aires: Siglo XXI Editores. Mollis, M. (2003). Un breve diagnóstico de las universidades argentinas: identidades alteradas. Las universidades en América Latina: ¿reformadas o alteradas? La cosmética del poder financiero (pp. 203-216). Consultado el 28/02/16 en: http:// s3.amazonaws.com/academia.edu.documents/31031289/11mollis.pdf?AWSAccessKeyld=AKIAJ56TQJRTWSMTNPEA\&Expires=1478546369\&Signature=DYplk49G8xtq9\%2BKgUxvah64A44k\%3D\&response-content-disposition=inline\%3B\%20filename\%3DUn_breve_diagnostico_de_las_universidade.pdf Unión Latinoamericana de Extensión Universitaria (ULEU) (2011). Declaración de la ULEU, Santa Fe. Consultado el 08/02/16 en: https://www.google.com.ar/ url?sa $=t \& r c t=j \& q=\& e s r c=s \&$ source=web\&cd=2\&cad=rja\&uact $=8 \& v e d=0$ ahUKEwjUzsK5pZfQAhVFFZAKHTB7AhMQFggfMAE\&url=http\%3A\%2F\%2Fwww.unl. edu.ar\%2Farticles\%2Fdownload\%2F5171\&usg=AFQjCNE3vPbZLoQAsOjvitQSNPz7nJkgeA\&sig2=SWi3kxOZTdFKhel5EdKn1w\&bvm=bv.137904068,d.Y2। 